Journal of Information and Communication Engineering (JICE), 6 (1): 318-320, June 1, 2020

e-ISSN: 2432-5465, p-ISSN: 2186-9162

Applied Science and Computer Science Publications

\title{
Development of Drone Detecting Free Parking Space for Car Parking Guidance
}

\author{
Tasato Yuske \\ Department of Media \\ Information Engineering \\ National Institute of \\ Technology, Okinawa College
}

\author{
Zacharie Mbaitiga \\ Department of Media Information \\ Engineering National Institute of \\ Technology, Okinawa College, \\ Okinawa, Japan \\ E-mail: zacharie@okinawa-ct.ac.jp
}

\begin{abstract}
This paper outlines the drone detecting free parking space for car parking guidance in order to reduce the work load of parking guardman. For this guidance system be effective we focused first on the white line edge of the car parking free space using CMOS cameras mounted in front and under Parrot airdrome 2.0 to capture the image. The input image taken from the camera was processed in the OpenCV software environment. The white line edge of the free parking space was detected first in RBG color, then converted to HSV. And finaly a mask processing was performed based on the outline information of the white line. Next the range in which the presence or absence of the car in the parking space was performed using template matching based on the data set taken from the School parking lot. Algthouh the experimental result shows a good result in which the drone was able to detect the free space in the parking lot the software still need improvement.
\end{abstract}

Keywords: Drone; ardrone 2.0; template matching; parking lot

\section{INTRODUCTION}

$\mathrm{W}$ e are living in a society where almost 2 person over three have cars in a developed countries and 1 person over three in an under developed countries. These data show without doubt that cars are the principal means of transportation in our modern society. Having a car require having a parking space to park the car and that is not a big issue for particular. But as for the super markets and shopping malls or anywhere holydays event are organized it does not require not only a big parking space or parking lot for customers but how to deal with the car guidance issue for many cars coming over and over all days. To deal with this issue most of the shopping malls and super markets and event organizers usually hire a traffic security guards to orient the incoming car to the designate parking lot and the number of the guards usually is so limited for cost saving. So increasing the number of guards will increase the cost as well. Due to the lack to the sufficient car guards about $30 \%$ of traffic accidents are occurred in parking lots. This is caused by irregularity movement of cars in the parking lot when the driver sees a free parking space and rush to park his/her car to avoid another driver to take a lead. If a car can be guided directly to a free parking space using drone, the number of the collision in the parking lot can be reduced. To the end in this research, we proposes a drone detecting a free parking space through image processing for car guidance.

\section{II.METHODOLOGY}

\section{A. Gray Sclae image conversion}

The following are the process of the free parking space detection where we have first focused on the parking free space white line from the inputted image of the camera (Fig.1). The white line here means the free space border lines. Since the white line color is basically the target to be detected, the detection is done through the performance of the binarization image processing

Corresponding Author:

Zacharie Mbaitig

Okinawa College, Japan. E-mail: zacharie@ okinawa-ct.ac.jp 
which started by the conversion of the inputted image to gray scale followed by binarization process as shown in Fig.2. For the binariation image we have set the threshold value to 187 then converted the pixel value below the threshold value to 0 representing the black color and the pixel value above the threshold value to 255 for the white color (Fig.3)

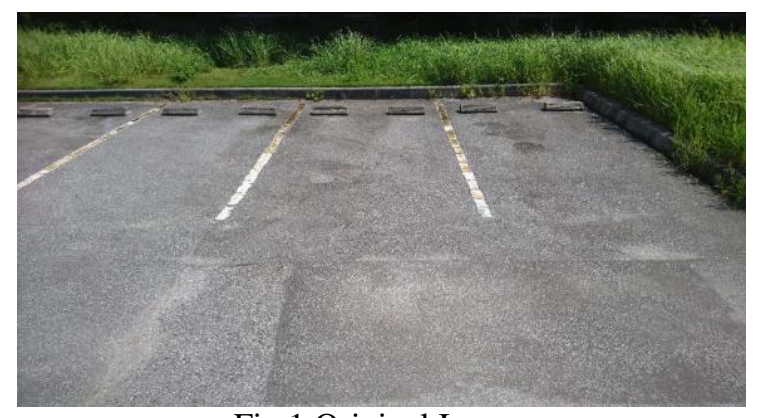

Fig.1 Original Image

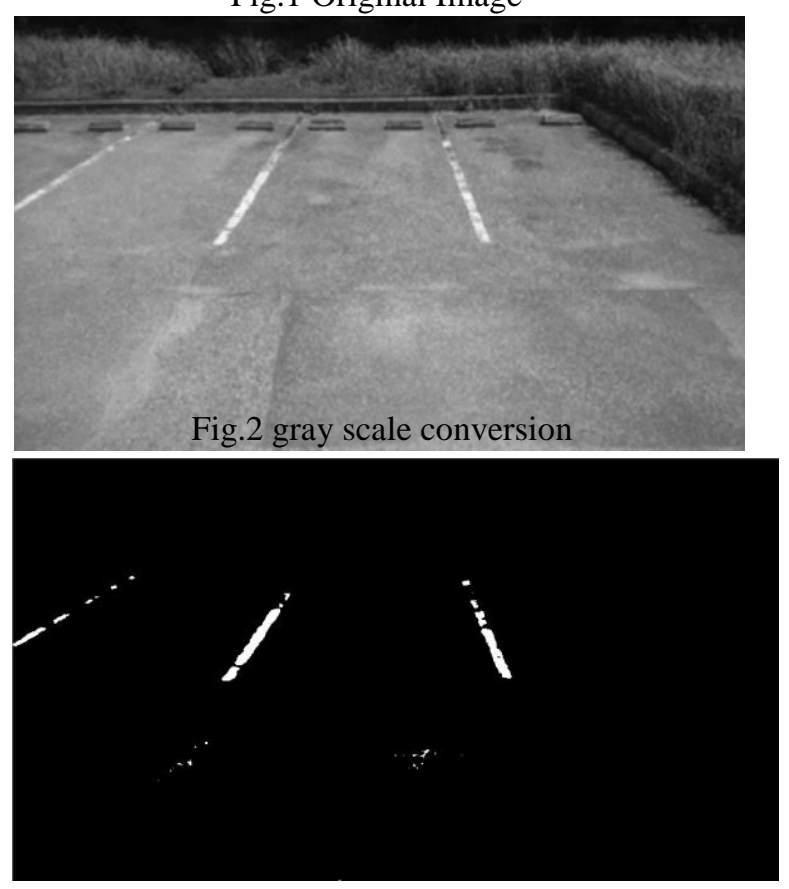

Fig.3 Binarization

But after the binarization processing we have found that the white lines surrounding the free parking space cannot be entirely detected by only binarization image processing due to aging deterioration. Therefore the need to cover the RGB image to HSV in order to solve the area of discoloration seen during the binarization process.

\section{B. Detection of white line discoloration}

The detection of the discoloration section of the white line was done through the conversation of the RGB color to HSV. Here $\mathrm{H}$ stands for Hue, $\mathrm{S}$ for saturation and $\mathrm{V}$ for lightness respectively. Following are the minimum and maximum value of the HSV we have set.

Minimum value of Hue is set to 10

Maximum value of Hue is set to 25

Minimum value of the Saturation is set to 32

Minimum value of the lightness is 110 respectively

Once the conversation is completed and process the image we then applied the filtarization in order to remove noises from the image to facilitate the recognition. Below are the combination result of the two image processing (Fig4) and the image with free noise (Fig.5).

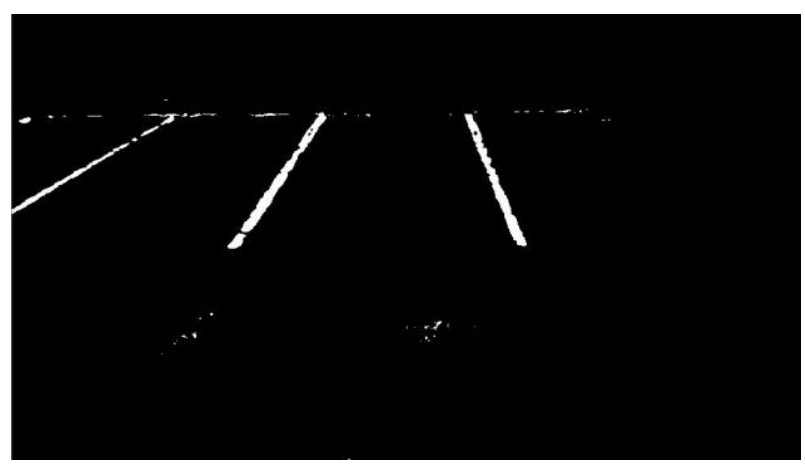

Fig.4 Combination of the binarized image and HSV

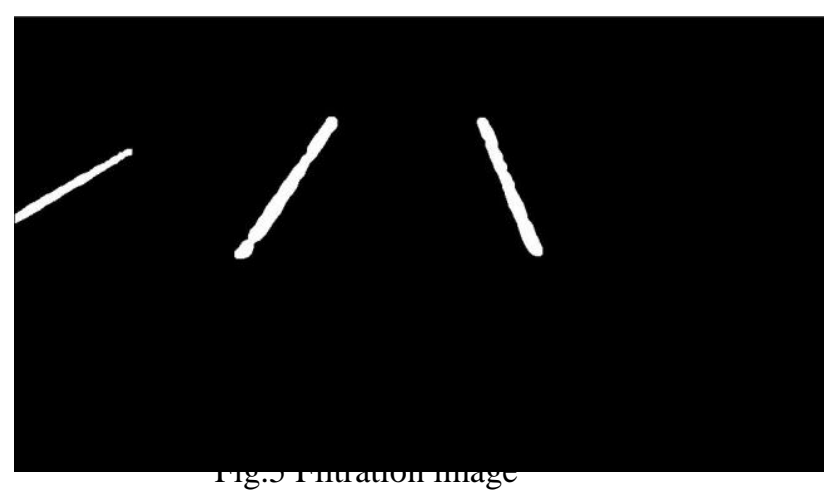

After we have removed all noises from the image, we moved on onto the next step which is the recognition process using the image processing template matching 
method. Following is the referred equation to the process.

$$
\begin{gathered}
I_{1}(A, B)=\sum_{i=1 \ldots 7}\left|\frac{1}{m_{i}^{A}}-\frac{1}{m_{i}^{B}}\right| \\
m_{i}^{A}=\operatorname{sign}\left(h_{i}^{A}\right) \cdot \log h_{i}^{A} \\
m_{i}^{B}=\operatorname{sign}\left(h_{i}^{B}\right) \cdot \log h_{i}^{B}
\end{gathered}
$$

Where:

$$
\begin{aligned}
& \text { A }=\text { image object } 1 \\
& \text { B }=\text { image object } 2 \\
& h_{\tilde{f}}^{A}=\text { moment of Hue A } \\
& h_{\tilde{f}}^{B}=\text { moment of Hue B }
\end{aligned}
$$

This work proposes the development of drone detecting free parking space for car parking guidance where the experimental results proved the merit of the method we have used as the exact expected result have been obtained. Although some misdetection has been detected when the experiment test goes on the whole detection rate is about $90 \%$. The next step is to find out what is the cause of the misdetection and improve the software again by paying attention to the match score and other criteria.

\section{Acknowledgement:}

This research is partially supported by SCOPE (Strategic Information and Communications R\&D Promotion Program), the Ministry of Internal Affairs

\section{REFERENCES}

[1] Mabitiga Zacharie, Satoshi Fujii, Shimoji Minori, "Rapid Human body Detection in Disaster Sites using Umage Processing from Unmanned Aerial Vehicle Cameras," Journal of Advanced Control, Automation and Robotics(JACAR), Applied Science and Computer Scoence publications vol. 3(2), pp. 96-101, December 29, 2017

[2] http://opencv.jp/opencv-2svn/cpp/index.html> 2016.9.28

[3] The general Insurance Association of japan. http://www.sonpo.or.jp/en/

[4] OpenCv $2.2 \mathrm{C}++$ open source http://opencv.jp/opencv2svn/cpp/

[5] puku0x 「CV Drone ( OpenCV + AR.Drone) $\rfloor$ <https://github.com/puku0x/cvdrone> 2016.9.28
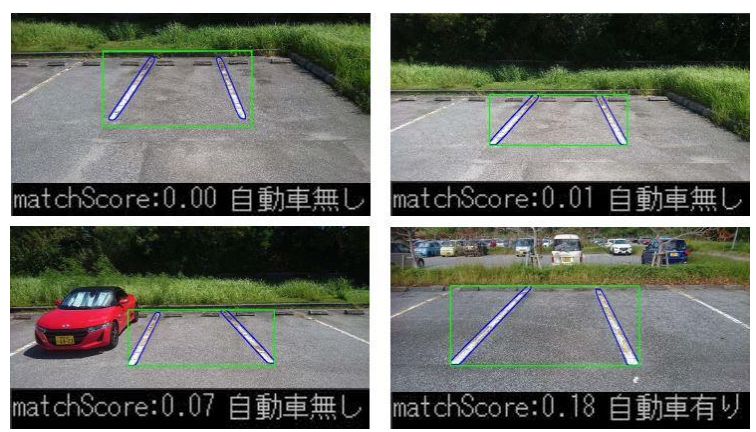